\title{
RESISTENCIA ANTIDESARROLLISTA Y EMERGENCIA DE MOVIMIENTOS SOCIALES DESDE LA ECOLOGÍA POLÍTICA: CASO DE LA SOJA PARA BIOCOMBUSTIBLES EN MATO GROSSO, BRASIL
}

\author{
Mauricio López Oropez \\ Facultad Latinoamericana de Ciencias Sociales, FLACSO-ECUADOR \\ Mauricio Calpa Burbano \\ Facultad Latinoamericana de Ciencias Sociales, FLACSO-ECUADOR
}

Resumen.- La creciente y descontrolada tendencia actual de cultivo agrícola de soja destinada al procesamiento y producción de biocombustibles está generando impactos (externalidades) de profundo impacto negativo para las comunidades rurales de campesinos e indígenas en el estado de Mato Grosso, Brasil, así como para toda la región continental, en términos de incremento de la pobreza, exclusión en cuanto a tenencia de la tierra, fuertes efectos ecológicos negativos en la reserva natural más importante del mundo: La Amazonía, y otros más, desencadenando en la emergencia de movimientos socio-políticos de resistencia contrahegemónica.

\begin{abstract}
The increasing, and out of control, tendency towards agricultural cultivation of soybean, destined to process and produce biofuels, is generating deep negative impacts for the rural communities of peasants and native-indigenous population in the region of Mato Grosso, Brasil, as well as for the south American region, in terms of increasing poverty, exclusion due to unequal ownership of land, strong negative ecological effects in detriment of the Amazonian reserve, and others, provoking the emergence of contrahegemonical and resistance socialpolitical movements.
\end{abstract}

\section{Introducción y antecedentes}

La presente reflexión la hemos realizado a partir del análisis de un tema de profunda importancia y vigencia para todos los sectores sociales, y en general para todos aquellos que se están haciendo preguntas de fondo con respecto a la crisis humana actual, y el futuro de la misma.

El tema de la ecología es un tema ineludible en cualquier aproximación a las problemáticas fundamentales actuales, y en la dinámica de relaciones de poder y la aparición de nuevos sistemas sociales que se están gestando alrededor de este componente esencial de nuestra construcción como humanidad. Este trabajo se centra especialmente en los aportes de la ecología política para 
hacer una crítica del modelo capitalista de desarrollo prevaleciente, y de su impacto sobre los procesos locales de los países del llamado tercer mundo.

Nos centraremos en la creciente demanda energética mundial, y el "boom" de los mercados alternativos para la producción de biocombustibles (biodiesel), para analizar, de entre la enorme cantidad de consecuencias negativas que acarrea, los impactos en los temas ambientales y sociales, analizados específicamente en Brasil, en el estado de Mato Grosso; territorio de mayor producción y crecimiento de soja a nivel mundial, además de ser región de frontera con la reserva ecológica más importante a nivel mundial: la Amazonía.

La problemática de dominación y exclusión alrededor del modelo capitalista, y la explotación que ejerce sobre los países llamados tercer mundistas, tiene una larga historia detrás, y es en esta lógica de relaciones de poder en la que se circunscribe nuestra reflexión.

Cuando los europeos colonizaron por primera vez el mundo no europeo, consideraban que su deber era descubrir y conquistar, someter, ocupar y poseer. Parece ser que las potencias occidentales todavía se mueven por este impulso colonizador de descubrir, de adueñarse, y de poseer todo, todas las sociedades, y todas las culturas. Las colonias se han ampliado ahora a los espacios interiores (Shiva 2001: 21).

Aunque la lógica colonizadora ha cambiado de forma, hoy la dinámica de acumulación sigue presente como proceso de dominación de los grupos más vulnerables y se ha extendido a la frontera de la ecología donde se pretende una dominación de los recursos en un nivel de explotación insostenible por la limitada "capacidad de carga" de nuestro planeta. Ante esta situación se pone en cuestionamiento todo modelo de consumo (sobre todo el de energías no renovables y renovables). "Hoy el hombre sigue siendo, más que nunca, el enemigo del hombre, no sólo porque sigue entregándose como nunca a la matanza de sus semejantes, sino también porque sierra la rama donde está sentado; el medio ambiente" (Castoriadis 2006: 279).

La experiencia del planeta cuenta con millones de años en su proceso de constitución como sistema integrado, y su relación con todas las especies vivientes ha sido el marco de autonomía y regulación que ha dado lugar a la vida en todas sus expresiones. Hoy la capacidad de "autoorganización" del planeta está en cuestionamiento y, ante los efectos del estilo de vida capitalista actual, también su capacidad de "autosanación" está en predicamento (Shiva 2001).

El problema ecológico se ha vuelto sobre todo político, y las lógicas justificadoras de una economía ecológica quedan en segundo plano, ya que científicos de distintas ramas expresan que el modelo de consumo y explotación actual no es sostenible al no encontrar una armonía conciliadora con el medio ambiente, a menos que se den cambios rotundos. "Tomar en 
cuenta el medio ambiente, el equilibrio entre la humanidad y los recursos del planeta es una evidencia central para toda política verdadera y seria" (Castoriadis 2006: 274).

\section{Marco Teórico "Ecología Política": Explotación ambiental y su efecto en las condiciones de vida de los sectores más pobres (rurales e indígenas).}

Reconocemos en la ecología política un recurso y una propuesta que nos permitirá analizar una realidad concreta regional-territorial, ya que nos permite integrar los fenómenos que circundan el papel del modelo capitalista frente a las realidades sociales y medioambientales de un grupo humano específico, así como las respuestas desde la resistencia comunitaria que permiten encontrar caminos contrahegemónicos frente al esquema explotador.

La ecología es subversiva porque cuestiona el imaginario capitalista que domina el planeta. Ella recusa su motivo central según el cual nuestro destino es aumentar sin cesar la producción y el consumo. Muestra el impacto catastrófico de la lógica capitalista en el medio ambiente natural y en la vida de los seres humanos (Castoriadis 2006: 265).

La ecología política es reconocida como un nuevo espacio de choque de dinámicas sociales; no es simplemente una reinterpretación con nuevas herramientas: es una posibilidad inédita de responder a una situación extrema igualmente inédita; "implica desbrozar el terreno, dislocar las montañas conceptuales y movilizar el arado discursivo que conforman su suelo original para construir bases seminales que den identidad y soporte a este nuevo territorio" (Leff 2006: 21).

Es importante retomar lo que plantea Vandana Shiva (2001) con respecto a la propiedad de la biodiversidad, donde argumenta que ésta es un recurso que no puede ser insertado en una lógica de mercado, ya que tiene una propiedad colectiva por la existencia de un tejido comunitario que vela por su reproducción, y se relaciona con ésta a través de una ética del respeto. Las comunidades rurales, especialmente de campesinos indígenas en América Latina, han mostrado en diversas experiencias su capacidad para relacionarse con el medio ambiente de manera más armónica, tener un beneficio mesurado de éste, y ser capaces de cuidarlo para su reproducción; una relación de genuina reciprocidad.

Este esquema ha cambiado por el fuerte peso de la lógica de mercado, por las condiciones de desigualdad para muchos productores que tienen qué intensificar el uso del suelo para sobrevivir (aplicando además agentes externos de la revolución verde que afectan fuertemente el equilibrio del sistema), y sobre todo por la presencia de intereses industriales que hacen de 
la tierra el campo ideal de explotación actual para ampliar sus ganancias. Esto es especialmente cierto en el cultivo de productos relacionados con la elaboración de biocombustibles para responder a la demanda energética de los países del norte, como el caso de la soja en el estado de Mato Grosso, Brasil.

Muchos actores sociales están apareciendo, o haciéndose visibles ante esta situación, y están tomando parte en esta lucha de poderes, que es también una lucha de clases, donde la ecología política es el espacio social donde "se están construyendo nuevas identidades culturales en torno a la defensa de las naturalezas culturalmente significadas [...] Estas identidades se han configurado a través de luchas de resistencia, afirmación y reconstrucción identitaria frente a procesos de apropiación y transformación de la naturaleza inducidos por la globalización económica" (Leff 2006: 26).

Integraremos en nuestro análisis la concepción de "ecologismo de los pobres" planteada por Martínez Alier (2004), siendo ésta una rama de la ecología política que pone especial énfasis en la situación de los países pobres del sur y sus recursos naturales, los cuales se mantienen unidos a los países del norte por la dependencia de los primeros en el tema energético y de materias primas. En esta dinámica norte-sur se dan fuertes relaciones de poder basadas en la coerción y presión sobre recursos naturales y materias primas en función de las grandes deudas existentes de parte de los países pobres, con respecto de los ricos.

\section{Impactos de la explotación ambiental}

A pesar de que no existe un estándar único que defina los efectos del modelo económico capitalista actual sobre el medio ambiente, hay un sinfín de aportes científicos que han generado índices de medición que dan cuenta de la gravedad del asunto ${ }^{1}$; especialmente si los confrontamos con la realidad de que Estados Unidos, Europa, y ahora China, han incrementado su demanda de materia prima, sobre todo la energética, de manera exponencial en las últimas décadas, y con el hecho de que la mayor parte de éstas provienen de la importación desde los países pobres; países que cuentan con una gran riqueza en biodiversidad, y están especializados en la extracción estas materias. "La desigual incidencia de los daños ambientales no ya frente a otras especies o frente a las generaciones futuras de humanos, sino en nuestra propia época, da nacimiento al ecologismo popular o ecologismo de los pobres" (Martínez Alier 2004: 81).

Contamos también con la claridad del aporte de O'Connor (2002) quien plantea la insostenibilidad del modelo actual a partir de lo que llama la "segunda contradicción del capitalismo", la cual se refiere a la incompatibilidad que existe

\footnotetext{
${ }^{1}$ Para profundizar en fundamentos teóricos y técnicos proponemos la lectura del Capítulo III. Índices de (in)sustentabilidad y neomaltusianismo en el Texto "El ecologismo de los pobres" de Joan Martínez Alier
} 
entre la feroz avidez de un incremento de ganancias de parte del modelo capitalista y la limitada, y cada vez más reducida, "capacidad de carga" del medio ambiente. Latouche (2003) a su vez expresa que este modelo de sociedad no es deseable incluso para los más ricos, ya que el tipo de condiciones de vida, especialmente medioambientales, les afecta de igual manera y pone en riesgo su existencia, al igual que la de toda la humanidad. Una vez más la necesidad de una transformación de fondo aparece como elemento ineludible.

\section{Impactos sociales de la exp lotación a mbiental en la vida de l os más pobres}

El efecto directo de esta lógica de consumo, y su impacto en los sectores sociales más vulnerables, es evidente, "existen casos en los que la resistencia y las culturas locales han sido destruidas junto con los ecosistemas locales" (Martínez Alier 2004: 69). Los grupos sociales que han estado siempre más vinculados a la tierra son los indígenas y/o grupos campesinos rurales, quienes fueron rechazados por el modelo urbano de desarrollo por mucho tiempo al ser vistos como simples proveedores de productos básicos. Hoy sus espacios, e incluso sus conocimientos y prácticas, son del mayor interés para las industrias de los biocombustibles (y de las farmacéuticas) en su intención utilitaria y de acumulación.

Hoy estos grupos están siendo despojados de sus tierras, condenados a ser proletarios con condiciones laborales ínfimas en las grandes industrias, y bajo el efecto de agentes externos (pesticidas, fertilizantes, etc.) para incrementar la productividad, teniendo fuertes impactos en su salud. Hoy están viviendo una nueva dinámica de lucha de clases pues, además de la defensa de la tierra, son estos grupos quienes están emprendiendo la defensa del medio ambiente de la mano de ONG's locales e internacionales. "Los impactos de estos cambios caen desproporcionadamente sobre algunos grupos sociales que muchas veces protestan y resisten. Algunos grupos amenazados apelan a los derechos territoriales indígenas y también a la sacralidad de la naturaleza para defender y asegurar un sustento" (Martínez Alier 2004: 27).

El tema de la soja transgénica es especialmente importante, ya que se están desplazando las fronteras agrícolas, sobre todo en la Amazonía, para satisfacer la creciente demanda energética mundial, y, como veremos en el apartado siguiente, las grandes industrias multinacionales de los biocombustibles están convirtiendo regiones enteras, como Mato Grosso al suroeste de Brasil, en los grandes complejos dispuestos a satisfacer la enorme demanda mundial de soja tomando parte de la jugosa ganancia que significa este mercado. 


\section{Contexto: Boom de I os bi ocombustibles e $\mathrm{n}$ I a I ógica de c onsumo energético mundial, y en el estado de Mato Grosso, Brasil. Caso de la soja para biodiesel.}

La humanidad se mueve a través del consumo de energía, se podría decir que la primera fuente de energía que conoce la humanidad es la biológica que proviene de las plantas, luego, la evolución del hombre y su desarrollo desenfrenado lo llevó a crear máquinas que necesariamente tendrían que funcionar con energía, encontrando la fuente para ello en los combustibles a base de hidrocarburos como el petróleo. Históricamente esto es lo que ha contribuido a la evolución de la humanidad generando centros de producción y de consumo de petróleo. Entre las regiones que más consumen el combustible están "América del Norte y Asia del Pacifico quienes consumen el 90\% de la producción mundial de petróleo". ${ }^{2}$

Las grandes consecuencias que ha generado esta actividad para el planeta son incalculables, comenzando por lo que es hoy por todos conocido como el calentamiento global, y de ahí se desencadenan toda una serie de secuelas negativas para la vida de los seres humanos en general, sin entrar en detalle de todos estos problemas, pasamos a una gama de posibles soluciones, donde encontramos la tendencia actual a retomar la energía biológica de las plantas; lo que se conoce en la actualidad como los biocombustibles.

Pareciera que los biocombustibles son una alternativa viable para la mitigación de los desastres provocados por la explotación, producción y consumo de hidrocarburos. Estos biocombustibles pueden ser procesados en tres diferentes formas, sólidos, líquidos y gaseosos, los cuales pueden provenir de materia orgánica animal o vegetal. En este caso, se analizará la forma de biocombustible líquido, el cual se clasifica en dos productos; el bioetanol, que utiliza materias primas como la caña, el maíz, la yuca o papa, y el biodiesel que utiliza materias primas como, - aparte de las grasas animales - la palma, la colza, el girasol y la soja, entre otros.

El uso de biocombustibles, especialmente del biodiesel, se remonta al año $1900^{3}$, tendencia que ha venido incrementándose, siendo una proclamada alternativa viable desde el punto de vista ambiental y desde el punto de vista económico. Se presenta, sobre todo, como una alternativa rentable para la

\footnotetext{
2 "A ambas regiones se atribuye casi el $90 \%$ del incremento del consumo de petróleo en los últimos 10 años". http://www.bbc.co.uk/spanish/specials/1421 petroleo/page3.shtml [Consultado 03/16/09, 20:00pm]

3 "Siendo Rudolph Diesel, quien lo utilizara por primera vez en su motor de ignición compresión y quien predijera el uso futuro de biocombustibles. Durante la segunda guerra mundial, y ante la escasez de combustibles fósiles, se destacó la investigación realizada por Otto y Vivacqua en el Brasil, sobre diesel de origen vegetal, pero fue hasta el año de 1970, que el biodiesel se desarrolló de forma significativa a raíz de la crisis energética que se sucedía en el momento, y al elevado costo del petróleo". http://www.eco2site.com/informes/biodieselm.asp. [Consultado 031709 5:55pm]
} 
acumulación de capital, especialmente para los empresarios que se dedican a la producción y transformación de la materia prima para este producto; es así como su producción año tras año se viene incrementando, sobre todo en países donde se cuenta con las condiciones, tanto climáticas, como espaciales para el cultivo de grandes extensiones de materia prima para el procesamiento de este combustible; como es el caso de Brasil en América Latina, quien en el periodo 2006 - 2007 incrementó su producción en un 400\% (Cuadro No. 1).

Cuadro No. 1 Producción de Biodiesel en el Mundo (2006 - 2007) toneladas

\begin{tabular}{|c|l|r|r|r|c|}
\hline Rank. & \multicolumn{1}{|c|}{ País } & 2007 & 2006 & Diferencia & $\begin{array}{c}\text { Crecimiento } \\
07-06\end{array}$ \\
\hline 1 & Alemania & 2.000 .000 & 2.200 .000 & -200.000 & $-9,1 \%$ \\
\hline 2 & Estados Unidos & 1.200 .000 & 750.000 & 450.000 & $60,0 \%$ \\
\hline 3 & Francia & 1.150 .000 & 550.000 & 600.000 & $109,1 \%$ \\
\hline 4 & Italia & 550.000 & 500.000 & 50.000 & $10,0 \%$ \\
\hline 5 & Malaisia & 300.000 & 120.000 & 180.000 & \\
\hline 6 & Brasil & 300.000 & 60.000 & 240.000 & $400,0 \%$ \\
\hline 7 & España & 200.000 & 125.000 & 75.000 & $60,0 \%$ \\
\hline 8 & Reino Unido & 200.000 & 100.000 & 100.000 & $100,0 \%$ \\
\hline 9 & Colombia & 200.000 & 50.000 & 150.000 & $300,0 \%$ \\
\hline 10 & Indonesia & 164.000 & 1.000 & 163.000 & $16300,0 \%$ \\
\hline \multicolumn{2}{|r|}{ Total Top10 } & 6.266 .007 & 4.458 .006 & 1.808 .001 & $40,6 \%$ \\
\hline \multicolumn{2}{|r|}{ Total mundial } & 7.904 .000 & 5.416 .000 & 2.488 .000 & $45,9 \%$ \\
\hline
\end{tabular}

Fuente: F.O. Lichts. httb://www.biofuel2g.com/Ponencias/Carlos Fernandez.

Dentro de los variados cultivos que se pueden utilizar para producción de biodiesel, en Brasil se han incrementado las áreas para el cultivo de oleaginosas, especialmente el cultivo de la soja; ésta ha presentado una tendencia creciente en la siembra de cultivos desde los años 60 (Ver Anexo No. 1), acentuándose en la actualidad debido al incremento de la demanda energética mundial. Según un estudio realizado por el centro de monitoreo de los agrocombustibles "ONG Repórter Brasil":

[...] se estima que en el periodo 2007 - 2008 Brasil exportó 30.7 millones de toneladas de soja [...] el país asiste a una expansión casi ininterrumpida del área plantada con ese cultivo, que partió, [...] de la región meridional del país rumbo al Cerrado, y que en los últimos años mira con codicia las tierras donde está la floresta Amazónica. Con 21 millones de hectáreas de soja sembradas, lo equivalente a 45\% de toda el área cultivada en la [cosecha] 2007/08, la soja es, de lejos, el principal producto de la agricultura brasileña (Milani y otros, 2008: 5).

Ante una oportunidad tan rentable los complejos agroindustriales, tanto nacionales, como internacionales, establecieron sus empresas procesadoras 
(usinas). En la actualidad existen en Brasil "51 plantas procesadoras de biodiesel, en toda el área nacional con autorización de la Agencia Nacional de Petróleo (ANP) y se evalúan 50 proyectos para implementar nuevas plantas procesadoras de los cuales 16 incrementarán el complejo agroindustrial en el estado de Mato Grosso,[donde] la capacidad instalada de producción saltaría de los actuales 2,5 mil millones de litros al año para más de 4 mil millones" (Ver Mapa. Anexo, No. 2), (Milani et. al., 2008: 7).

Todas estas grandes concesiones, que aparentemente están generando "desarrollo" para algunos sectores de la población del estado Mato Grosso, han desencadenado una serie de impactos ambientales, económicos, agrarios y laborales en territorios y poblaciones rurales e indígenas que habitan en esas tierras tan codiciadas por el capital nacional y extranjero.

\section{Impactos socio-ambientales del cultivo de soja en Mato Grosso Brasil y los ef ectos en I a $v$ ida $d$ e I os $g$ rupos rurales $p$ obres ( campesinos $e$ indígenas).}

Como se ha mencionado existe una variedad enorme de impactos negativos relacionados con el cultivo de soja en el estado de Mato Grosso en Brasil. Por cuestión metodológica y limitación de espacio nos enfocaremos en los impactos ambientales sobre la biodiversidad Amazónica, así como en el impacto social sobre las comunidades rurales e indígenas de la región. En esta reflexión esbozaremos de manera crítica algunos de estos impactos para propiciar futuras reflexiones.

Comenzaremos presentando algunos de los obstáculos más significativos (Barkin 2002: 176) con los que se enfrentan los procesos sociales, los grupos rurales e indígenas, y ecologistas de la región, para consolidar una verdadera estrategia de Ecología Política para el manejo sustentable de los recursos:

\begin{tabular}{|l|l|}
\hline $\begin{array}{l}\text { Obstáculos m ás } \\
\text { sobresalientes } \\
\text { tomados de } \\
\text { Barkin (2002: 176) }\end{array}$ & $\begin{array}{l}\text { Análisis d e si tuación en el co ntexto M ato G rosso, } \\
\text { Brasil. Análisis realizado a partir del documento: "El } \\
\text { Brasil de los biocombustibles: Impactos de los Cultivos } \\
\text { sobre la Tierra, el Medio Ambiente y la Sociedad. SOJA- } \\
\text { Ricino 2008". Elaborado por Repórter Brasil. }\end{array}$ \\
\hline $\begin{array}{l}\text { Políticas } \\
\text { macroeconómicas y y } \\
\text { sectoriales } \\
\text { discriminatorias. }\end{array}$ & $\begin{array}{l}\text { a) El cultivo de soja transgénica demanda un uso intenso } \\
\text { y masivo de agroquímicos, los cuales tienen un efecto en } \\
\text { la fertilidad y humedad del suelo, en la contaminación de } \\
\text { mantos acuíferos, así como en la salud de los } \\
\text { trabajadores (Se habla de un incremento de incidencia }\end{array}$ \\
& $\begin{array}{l}\text { en cáncer para la región). } \\
\text { b) El gobierno regional, fuertemente vinculado a la } \\
\text { dinámica productora de soja, tiene una postura permisiva } \\
\text { para continuar la presión sobre la frontera Amazónica en } \\
\text { los enormes cultivos de soja de los grandes }\end{array}$ \\
\hline
\end{tabular}




\begin{tabular}{|c|c|}
\hline & $\begin{array}{l}\text { inversionistas en Mato Grosso. } \\
\text { c) Estancamiento en el cultivo de soja de los EEUU, lo } \\
\text { cual ha generado políticas más flexibles que promueven } \\
\text { el aumento en la producción, independientemente de sus } \\
\text { efectos colaterales, para proveer a dicho país, y al } \\
\text { mercado internacional, el deseado y rentable producto. } \\
\text { d) Brasil esperaría que el crecimiento de los próximos } \\
\text { años, especialmente desde Mato Grosso, esté } \\
\text { asegurado por la amplia demanda de la soja para } \\
\text { biocombustibles y otros usos. } \\
\text { e) Existen fuertes impulsos a la producción de soja, en la } \\
\text { región y en el país, con incentivos fiscales de parte del } \\
\text { Programa Nacional de Producción y Uso de Biodiesel. }\end{array}$ \\
\hline $\begin{array}{l}\text { Sistemas } \\
\text { inadecuados y } \\
\text { polarizados de } \\
\text { tenencia de la } \\
\text { tierra. }\end{array}$ & $\begin{array}{l}\text { a) La producción de soja transgénica requiere una } \\
\text { enorme concentración de la tierra para lograr la } \\
\text { competitividad deseada por el mercado de } \\
\text { exportaciones, provocando expulsión del hombre del } \\
\text { campo. } \\
\text { b) Los requerimientos para la competitividad del cultivo } \\
\text { de la soja están ampliando la explotación en tierras de la } \\
\text { Amazonía como estrategia para asegurar bajos costos } \\
\text { de la producción. } \\
\text { c) Se han dado repetidas experiencias de despojo de } \\
\text { tierras de campesinos, venta bajo coerción y a bajo } \\
\text { precio, y en ocasiones la muerte (desaparición) de } \\
\text { indígenas o campesinos ecologistas que se oponen a } \\
\text { esta situación. }\end{array}$ \\
\hline $\begin{array}{l}\text { Sesgo } \\
\text { anticampesino en } \\
\text { las instituciones de } \\
\text { desarrollo. }\end{array}$ & $\begin{array}{l}\text { a) La soja necesita enormes terrenos de cultivo para } \\
\text { garantizar su producción a gran escala, lo cual genera } \\
\text { una profunda tendencia al monocultivo, y a la vez un } \\
\text { rechazo de la propiedad campesina o indígena de cultivo } \\
\text { diverso. } \\
\text { b) Hay un control de stocks, de parte de los grandes } \\
\text { productores, para presionar los precios a su favor, ya } \\
\text { que generalmente es la misma empresa la que controla } \\
\text { los procesos de producción-adquisición, procesamiento y } \\
\text { exportación del producto. } \\
\text { c) Existe un claro rechazo a la presencia de grupos de } \\
\text { campesinos o indígenas con conciencia social y } \\
\text { ecológica, ya que son un verdadero problema para que } \\
\text { los grandes productores continúen su explotación de la } \\
\text { Amazonía en la región. }\end{array}$ \\
\hline $\begin{array}{l}\text { Distribución } \\
\text { desigual del ingreso } \\
\text { y el poder político. }\end{array}$ & $\begin{array}{l}\text { a) Blairo Maggi es a la vez Gobernador de la Provincia } \\
\text { de Mato Grosso y miembro de la familia con mayor } \\
\text { producción de soja del Brasil. } \\
\text { b) La mayor parte de la producción de soja está } \\
\text { relacionada con grandes haciendas de monocultivo y de }\end{array}$ \\
\hline
\end{tabular}




\begin{tabular}{|l|l|}
\hline & capital macro industrial, y los pequeños productores se \\
ven obligados a entrar en esta lógica de producción. \\
c) El monopolio de distribución de grandes consorcios \\
fijan precios extremadamente bajos para los pequeños \\
productores generando condiciones desiguales y dos \\
opciones: proletarizarse y trabajar en los cultivos \\
después de vender sus tierras a bajos precios, o migrar a \\
otro sitio.
\end{tabular}

\section{Impactos ambientales}

Lo fundamental en este aspecto es el peso de la explotación agroindustrial sobre la biodiversidad Amazónica, unido a las consecuencias que esto tiene en temas muy diversos, y que afectan en el presente, pero sobre todo lo harán en el futuro, a los ecosistemas regionales, e incluso continentales, además de los efectos en los grupos humanos relacionados con la región que se mencionarán posteriormente.

Sintomáticamente, la cosecha brasileña de soja de 2002, llegó a sus niveles record de 50 millones de toneladas, y los índices de deforestación llegaron paralelamente a su segunda tasa más elevada desde los años 1990, con $25.500 \mathrm{~km}$. [...] ]el cultivo de la soja se ha vuelto uno de los principales factores de deforestación. La desaparición del bosque amazónico plantea un problema grave, ya que casi toda la agricultura brasileña depende de su mantenimiento y 
de su papel de regulación de las lluvias en el centro-oeste y sudeste del país. Sin bosque, las principales regiones agrícolas del Brasil se volverán demasiado áridas para la producción de granos (Pasquis 2006: 52-53).

Pasquis (2006) también nos presenta datos reveladores sobre el impacto de la agroproducción de soja, especialmente la de Mato Grosso, sobre el cambio climático de la región y los efectos que esto acarrea para el equilibrio Amazónico, y todo el territorio (un territorio que no sólo responde al país Brasileño, sino a todos los países de dicha región del cono Sur). También nos presenta el efecto del monocultivo, con fuerte presencia de agentes químicos para la productividad, sobre el ciclo de las aguas.

Se habla de que hasta el 2004, 1 millón 200 mil hectáreas de la Amazonía fueron convertidos en plantaciones de soja (Milani et. al. 2008), y también se ha constatado que la vegetación nativa, con toda su diversidad, está siendo afectada por este monocultivo. Se han generado diversas iniciativas de conservación, pero los esfuerzos son muy limitados frente al gran agronegocio que se está abriendo alrededor de este monocultivo, e incluso se puede considerar que en las últimas giras internacionales del presidente Lula Da Silva, sus sesiones de trabajo han sido sobre todo con empresarios con capacidad de inversión en este jugoso negocio. El nivel de crecimiento de Brasil se ha visto, y se verá aún más, afectado por la crisis financiera mundial, así que intuimos que este emergente negocio será una estrategia central para sostener la economía nacional, aunada a la creciente demanda energética de los países ya mencionados.

\section{Impactos sociales}

En este apartado retomaremos una serie de factores vinculados al cultivo de soja que están teniendo un efecto negativo creciente en diversos ámbitos; sobre todo para los grupos rurales e indígenas del estado de Mato Grosso.

Cuando se hace una previsión sobre el futuro de la expansión de las áreas de soja en Brasil, no es difícil proyectar un agravamiento del nivel de concentración de la tierra en el país. El índice de Gini mide el grado de concentración - cero indica igualdad absoluta y 1 , concentración absoluta. Para Brasil, el índice de distribución de renta es del orden del 0,6, y para la concentración agraria está sobre 0,8 [...]Los inmuebles con área superior a 2.000 hectáreas corresponden a sólo $0,8 \%$ del número total de inmuebles, pero ocupan el mismo 31,6\% del área total (Milani et. al. 2008: 26)

Estos datos revelan una dinámica de concentración de tierra que tiene un efecto directo en la precarización de las familias que habitan las regiones de mayor producción agroindustrial (mayoritariamente campesinos indígenas). Como se ha dicho, Mato Grosso es el foco de mayor crecimiento nacional y mundial en el tema de cultivo de soja, por lo tanto es previsible el incremento de pobreza para dichas poblaciones, y la presión para la incorporación de 
indígenas y campesinos a una dinámica proletaria en condiciones muy precarias. Otro punto ya mencionado es la expulsión de las poblaciones de sus territorios, ya que este cultivo particular requiere de muy poca mano de obra ${ }^{4}$, así que las familias no tienen más remedio que migrar, perdiéndose la presencia de muchos actores con conciencia social para la defensa de la Amazonía.

Un hecho observado en la región es que los pequeños agricultores están vendiendo sistemáticamente sus lotes de tierra a los agricultores inmigrantes oriundos, principalmente, de Rio Grande do Sul, Paraná y Mato Grosso. El precio de la tierra que queda cercana a carreteras subió de aproximadamente R\$ 50 para más de R\$ 1.000 por hectárea, entre 1997 y el 2008 (Milani et. al. 2008: 26).

Un efecto social colateral a esta dinámica de producción capitalista es la violencia que se ha generado en contra de los grupos rurales e indígenas ${ }^{5}$, y ONGs, que desde una postura ecológica, política y social, se han enfrentado a los agroexportadores y gobiernos, que en el caso de Mato Grosso son la misma cosa, para denunciar el daño ambiental y la violación de derechos humanos y laborales acarreados por esta posición rapaz de explotación para el cultivo de soja y producción de biocombustibles.

El mismo documento de "Repórter Brasil" (Milani et. al. 2008: 31) nos presenta las formas de amenaza más fuertes hacia los grupos tradicionales, especialmente indígenas, traídas por el cultivo de la soja en regiones Amazónicas como Mato Grosso:

a) Ocupación directa de tierras tradicionales ya aseguradas por el Estado Brasileño.

b) Degradación ambiental del entorno de territorios tradicionales, afectando a las comunidades.

c) Presión política o judicial contra la demarcación de tierras demandadas como tradicionales- y ya reconocidas por estudios socioantropológicos.

d) Ocupación consentida de tierras tradicionales ("colaboración").

\section{Movimientos s ocio-políticos que c onfrontan e sta $r$ ealidad ur gente- emergente}

En Brasil hay una fuerte presencia de movimientos sociales históricos relacionados con la lucha frente al modelo capitalista de desarrollo, y sus

\footnotetext{
${ }^{4}$ Los empleos directos en el cultivo de soja, en equivalente a trabajador/año, para cada 100 hectáreas fue de 2 trabajadores en este producto masivo. Cifras del año 2000 tomadas de Fundación Seade/Sensor Rural en Landeros (2008: 9)

5 Se habla de algunos asesinatos relacionados con la disputa por la tierra en Mato Grosso. http://www.jornada.unam.mx/2008/02/17/index.php?section=mundo\&article=026n1mun
} 
consecuencias sobre las poblaciones más pobres. Es conocido el fuerte papel de la Teología de la Liberación, a través de las Comunidades Eclesiales de Base, en los años 70's y 80's para emprender una lucha por la tierra, los derechos humanos y laborales, en diversas regiones del país; estos movimientos tienen todavía cierta presencia hoy, pero sobre todo han generado cuadros de líderes y agentes sociales con una fuerte conciencia socio-política y de clase. Existe también la fuerte presencia nacional del Movimiento de los Sin Tierra (MST), quienes buscan una reivindicación del campesinado, y quienes, aunque no son un grupo tradicionalmente agroecológico, han asumido el tema ambiental como punto central de su postura política y social, especialmente a partir de las experiencias del Foro Social Mundial, y por el fuerte impacto del tema del cultivo de soja con las dinámicas comunitarias campesinas e indígenas.

Hay una constante violación de derechos humanos alrededor del tema de explotación del medio ambiente. Bajo esta situación no es raro que el tema ambiental estalle bajo la forma de conflictos sociales. En los casos en que se manifiestan protestas ciudadanas, éstas son combatidas por el propio estado. A pesar de sus debilidades, el estado sigue siendo capaz de contener y encauzar las expresiones ciudadanas (cumpliendo el papel de "Estado tapón") (Gudynas 2002: 139).

Las luchas sociales de los grupos rurales e indígenas, además de ONGs ecologistas, están gestando una estructura más fuerte y articulada, la cual ha logrado convertirse en interlocutora a través de grupos como el MST, y otras instancias nacionales e internacionales, para incidir en políticas públicas, promover la resistencia de parte de los grupos de base, y logrando una mayor concientización a nivel regional, nacional, e incluso internacional de la problemática.

En Mato Grosso se van posicionando muchas de estas luchas y movimientos sociales, ya que es un bastión de suma importancia para el enfrentamiento actual entre los intereses agroindustriales y gubernamentales que impulsan el cultivo de soja para biocombustibles, y los grupos contrahegemónicos que cuestionan este sistema insostenible social y ecológicamente. "La ética del ecologismo de los pobres nace de una demanda de justicia social contemporánea. Esta corriente señala que muchas veces los grupos indígenas y campesinos han coevolucionado sustentablemente con la naturaleza. Han asegurado la conservación de la biodiversidad" (Martínez Alier 2004: 27).

\section{Conclusiones}

Hoy tenemos frente a nosotros, a partir del presente estudio desde la ecología política, una realidad de lucha social orquestada por los intereses del poder económico y político de los países desarrollados, quienes pretender perpetuar el sometimiento y dominación de los países en desventaja o subdesarrollados 
excluyendo, desterrando y masacrando a pueblos rurales e indígenas que se encuentran asentados en territorios con grandes ventajas para sus intereses. Estos territorios son fundamentales para seguir consolidando el régimen social y económico capitalista imperante, el cual a su vez ha suscitado lo que se conoce hoy como una profunda crisis ambiental y social.

A partir de nuestro planteamiento hipotético inicial podemos constatar que se están gestando una serie de procesos sociales alrededor de la dinámica agroindustrial de producción de soja en Mato Grosso, Brasil. Si bien es cierto que existe una fuerza profundamente estructurada y hegemónica en los tejidos de poder que se articulan a la fuerza hegemónica del mercado internacional (desde su demanda de biodiesel por parte de los países más industrializados como EEUU, Europa y China especialmente), y donde se evidencia el peso de un gobierno nacional y regional brasileño que sustenta la ruta para el crecimiento económico y la superación de la crisis mundial en este creciente mercado, también es claro ubicar una enorme contradicción dentro de esta lógica de explotación que está encontrando su punto de quiebre desde sus propias entrañas.

Esta contradicción es evidenciada y confrontada por los fundamentos de la ecología política, los cuales expresan que a partir de estos esquemas de abuso ambiental y social se dan, con gran fuerza y vigencia, las condiciones para una articulación orientada a emprender una opción de resistencia contestataria, y la emergencia de una nueva lucha de clases que pueda detener esta rapacidad que está teniendo ya consecuencias irremediables en todo el planeta, y especialmente en la región Amazónica de Brasil.

Es importante salir de la lógica local para orientar una búsqueda territorial más amplia donde se pueda integrar una conciencia social a partir de tres elementos fundamentales:

1. El aspecto ecológico que ya no puede sostener la carga impuesta por este esquema de explotación utilitaria y excluyente, y que se ubica, en este caso de análisis, en la reserva de biodiversidad más importante para el mundo: La Amazonía.

2. El aspecto identitario de grupos campesinos, indígenas y rurales, que están sufriendo las graves consecuencias de esta dinámica capitalista generadora de mayor pobreza, exclusión y expulsión.

3. La violencia y las condiciones de explotación que se están generando alrededor de la producción de soja y otros productos como maíz, caña, etc. para la producción de biocombustibles, provocando además el alza de precios de productos básicos, y la represión o eliminación de cualquier movimiento o grupo que se muestre como opositor de esta posición fundamentada en el capitalismo. 
Ante todo esto se hace posible repensar una conciencia mundial desde las bases que pueda sustentarse en la ecología política, para articular y territorializar las dinámicas contrahegemónicas, más allá de los intereses sectarios de circunscripciones locales y nacionales, logrando además visibilizar el efecto de exclusión hacia identidades indígenas y rurales, no sólo en la región referida, sino en todo el planeta. Esta conciencia, que consideramos ya se está gestando, podría generar modelos alternativos de economía que respeten las dinámicas ambientales y sociales, cuestionando la lógica de consumo del modelo de desarrollo capitalista, especialmente el energético, y promoviendo una nueva visión que integre todos estos argumentos a la luz de la gran crisis financiera y ecológica que está cuestionando el esquema ideológico y estructural del desarrollo capitalista.

Para dar pasos firmes y bien sustentados hacia este horizonte recurrimos a las etapas que plantea Latouche (2008: 24), para alcanzar una sociedad sostenible: 1. Cambiar de valores y conceptos, 2. Cambiar de estructuras, es decir de sistema, 3. Relocalizar la economía y la vida, 4. Revisar nuestros modos de uso de los productos, 5. Responder al reto específico de los países del Sur, y 6 . Asegurar la transición de nuestra sociedad de crecimiento a la sociedad de decrecimiento.

\section{Bibliografía}

Alimonda, Héctor (2003). "Ecología Política: Naturaleza, sociedad y utopía". CLACSO. Buenos Aires.

(2006). "Los tormentos de la materia: Aportes para una ecología política latinoamericana”. CLACSO. Buenos Aires.

Castoriadis, Cornelius (2006). "Una sociedad a la deriva". Katz Editores. Buenos Aires.

Falconí, Fander, Hercowitz, Marcelo y Muradian, Roldán, eds. (2004). "Globalización y desarrollo en América Latina". FLACSO - Sede Ecuador. Quito.

Gudynas, Eduardo (2002) "La ecología política de la integración: reconstrucción de la ciudadanía y regionalismo autónomo" En Ecología Política: Naturaleza, sociedad y utopía, Ed. Héctor Alimonda p. 137 a 152, CLACSO.

Guha, Ramachandra y Martínez Alier, Joan (2000). "Varieties of Environmentalism". Earthscan. Londres.

Guimaraes, Roberto P. (2006). "Desarrollo sustentable en América Latina y el Caribe: desafíos y perspectivas a partir de Johannesburgo 2002" En Los tormentos de la materia, Ed. Héctor Alimonda, p. 87 a 114, CLACSO. 
(200). "La ética de la sustentabilidad y la formulación

de políticas de desarrollo" En Ecología Política: Naturaleza, sociedad y utopía, Ed. Héctor

Alimonda p. 53 a 82, CLACSO.

Harnecker, Marta (2002). "Sin tierra: Construyendo movimiento social". Siglo XXI Editores. Madrid.

Lapitz, Rocío y Gudynas, Eduardo (2004). "Los claroscursos del cultivo de soja en Mato Grosso". Observatorio del Desarrollo. Centro Latinoamericano de Ecología Social. CLAES.

Latouche, Serge (2008). "La apuesta por el decrecimiento". Icaria Editores. Barcelona.

Leff, Enrique (2006). "La ecología política en América Latina un campo en construcción" En Los tormentos de la materia, Ed. Héctor Alimonda, p. 21 a 40, CLACSO.

Martínez Alier, Joan (2004). "Ecologismo de los pobres". Icaria Editorial. Barcelona.

(2006). "Economía Ecológica y Política Ambiental". Fondo de Cultura Económica. México.

Milani, Aloísio, Mauricio Monteiro Filho, Spensy Pimentel y Verena Glass (2008). "El Brasil de los agrocombustibles: Impactos de los cultivos sobre la Tierra, el Medio Ambiente y la Sociedad. Soja-Ricino 2008".

Repórter Brasil. http://www.reporterbrasil.com.br/ [Consultado 12/03/09]

Naciones Unidas-FAO y Comisión Económica para América Latina-CEPAL (2007). "Oportunidades y riesgos del uso de la bioenergía para la seguridad alimentaria en América Latina y el Caribe". FAO y CEPAL.

http://www.rlc.fao.org/prior/segalim/bioenergia.htm [Consultado 12/03/09]

ONU Energía (2008). "Bioenergía sostenible: Un marco para la toma de decisiones". Naciones Unidas-FAO.

http://www.rlc.fao.org/es/prioridades/bioenergia/pdf/biocombustible.pdf

[Consultado 12/03/09]

O’Connor, James (2002). “¿Es posible el capitalismo sostenible?” En Ecología Política: Naturaleza, sociedad y utopía, Ed. Héctor Alimonda p. 27 a 52, CLACSO. 
Pasquis, Richard (2006). "Mercado y medio ambiente: el caso de la soja en la Amazonía Brasileña". Íconos Revista de Ciencias Sociales No. 24: pp. 47-56. FLACSO Sede Ecuador. Quito.

Schmink, Marianne y Wood Charles H. eds. (1991). "Frontier expansion in Amazonia". University of Florida.

Shiva, Vandadna (2001) "Biopiratería: El saqueo de la naturaleza y del conocimiento". Icaria Editores. Barcelona.

Stonich, Susan C. (1993). "I am destroying the land". Westview Press.

\section{ANEXOS}

\section{Evolución de los rendimientos de soya en Brasil,} (1961-2004)

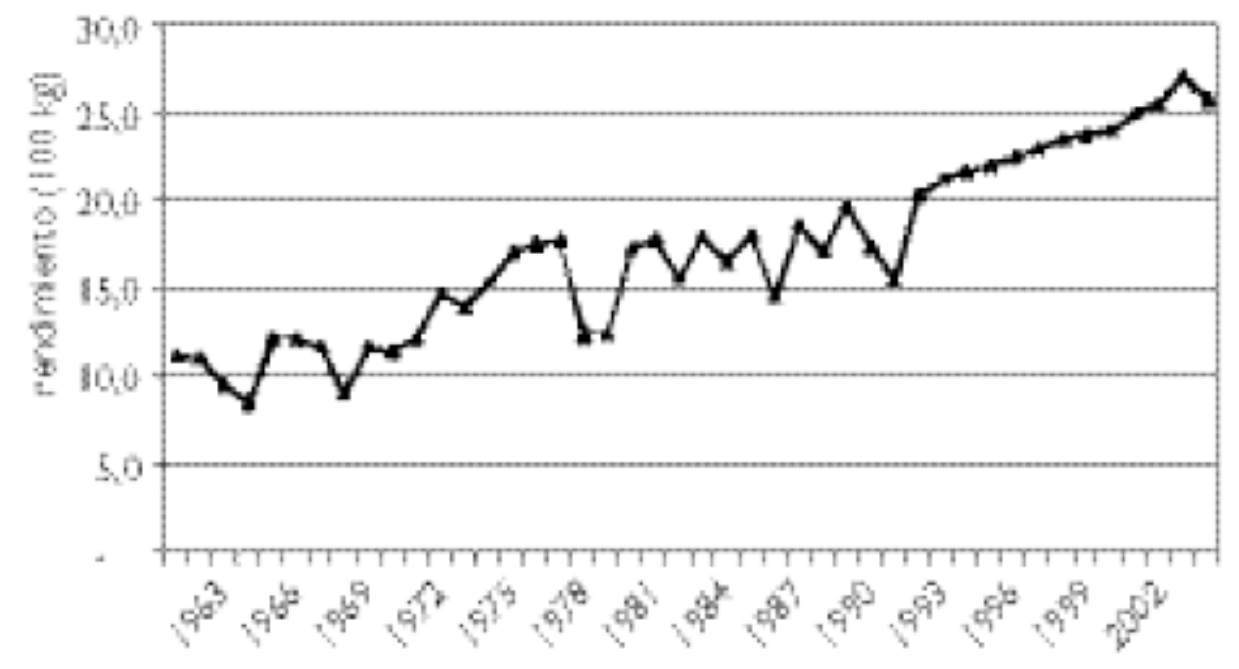

Fuente: Revista de Ciencias Sociales. ICONOS. Núm. 24, Quito, mayo 2006, pg. 49, .FLACSO - ECUADOR. 
ANEXO. No. 2

Incremento De C ultivos de Soja. Estado De Mato G rosso B rasil. (1996 2006)

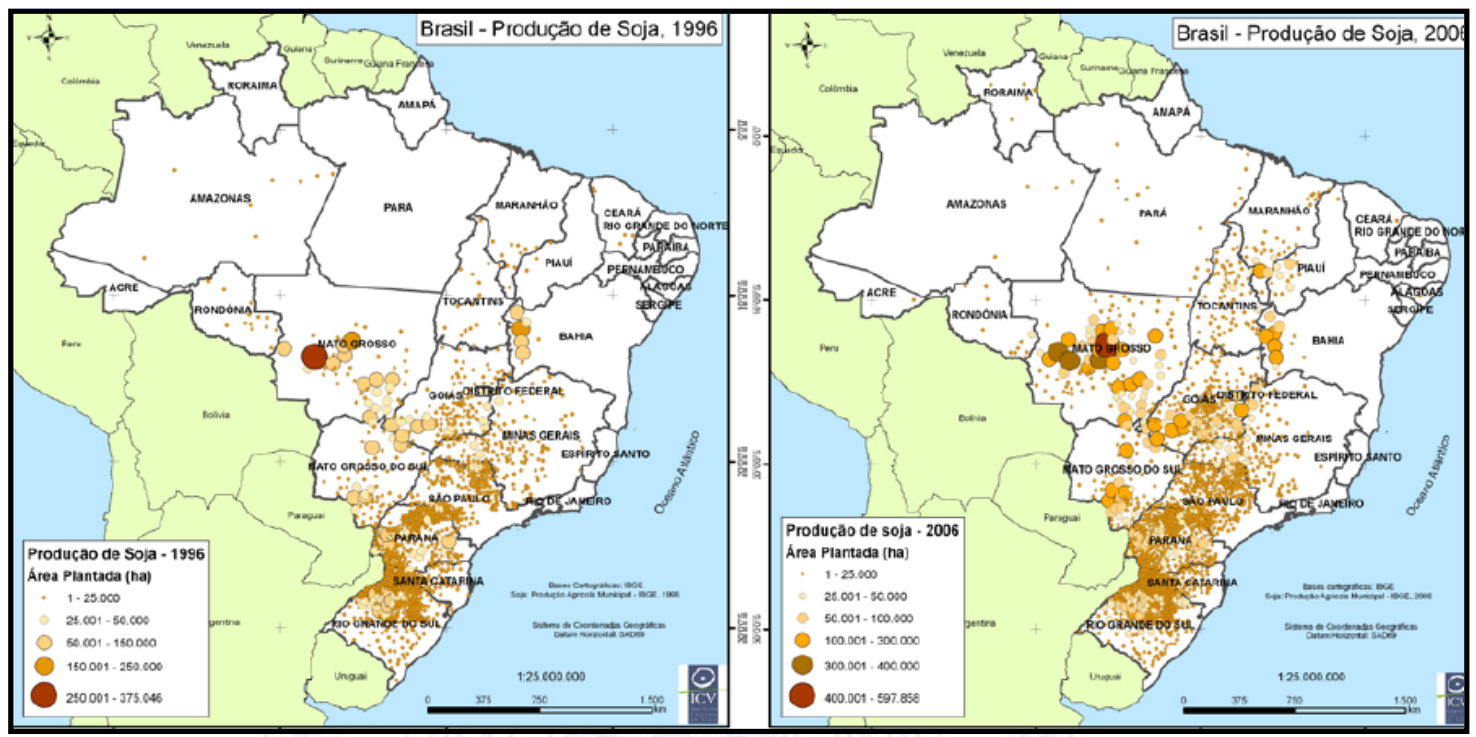

Fue: Reasil (Milani et. al., 2008: 7). http://www.reporterbrasil.com.br 